\title{
Electrorheological Rayleigh-Taylor instability at the interface between a porous layer and thin shell with poorly conducting couple stress fluid
}

\author{
N. Rudraiah ${ }^{\mathrm{a}, \mathrm{b}, *}$, Milan Kalal $^{\mathrm{c}}$, G. Chandrashekara ${ }^{\mathrm{a}}$ \\ ${ }^{a}$ UGC-Center of Advanced Studies in Fluid Mechanics, Department of Mathematics, Bangalore University, Central College Campus, Bangalore-560 001, India \\ ${ }^{\mathrm{b}}$ National Research Institute for Applied Mathematics (NRIAM), \#429/G, 7th cross, 7th Block (West), Jayanagar, Bangalore-560 070, India \\ ' School of Applied and Engineering Physics, Faculty of Nuclear Sciences and Physical Engineering, Czech Technical University in Prague, Czech Republic
}

\section{A R T I C L E I N F O}

\section{Article history:}

Received 5 December 2009

Received in revised form

8 April 2010

Accepted 14 July 2010

\section{Keywords:}

Electrorheological fluid

Couple stress

Inertial fusion energy

Laser radiation

Transverse electric field

Maximum growth rate

\begin{abstract}
A B S T R A C T
This paper is concerned with the study of the Electrorheological Rayleigh-Taylor instability (ERTI) at the interface between a densely packed saturated poorly conducting couple stress porous layer accelerated by a lighter poorly conducting couple stress fluid in a thin shell in the presence of a transverse electric field and laser radiation. A simple theory based on fully developed flow approximations is used to derive the dispersion relation for the growth rate of ERTI. The cutoff and the maximum wave numbers and the corresponding maximum frequencies are obtained. It is shown that the effects of couple stress parameter and the electric field reduce the growth rate considerably compared to a non-conducting fluid in the absence of an electric field. These are favorable to control the surface instabilities in many practical applications discussed in this paper.
\end{abstract}

(c) 2010 Elsevier Ltd. All rights reserved.

\section{Introduction}

In this era of globalization, liberalization, modernization, increase in population and industries require more electric power, particularly an uninterrupted power supply. The depletion of fossil fuel and hydroelectric power due to atmospheric vagaries have strained adequate power supply for overall development of a country. Therefore, there is an urgent need of power supply from the unconventional sources of energy which are economically affordable and environmental friendly. Therefore, many unconventional methods like nuclear power, solar power, wind power and so on have been proposed to generate electric power. The United Nations, through International Atomic Energy Agency (IAEA), is proposing to generate an adequate electric power from Inertial Fusion Energy (IFE) by fusing the two hydrogen isotopes Deuterium-Tritium (DT), in Inertial Fusion Target (IFT), which is also one of the environmental friendly and unconventional methods of generating electric power. The nuclear picture of DT is such that there will be a strong repulsive force between them which prevents the natural fusion of DT. Therefore, there is a need of external agency like laser radiation to fuse them. The high

\footnotetext{
* Corresponding author at: UGC-Center of Advanced Studies in Fluid Mechanics, Department of Mathematics, Bangalore University, Central College Campus, Bangalore-560 001, India.

E-mail addresses: rudraiahn@hotmail.com (N. Rudraiah), Kalal@fifi.cvut.cz (M. Kalal), chandrashekarg.4@gmail.com (G. Chandrashekara).
}

intensity laser radiation is impinged at the ablative surface of IFT to fuse DT. This radiation no doubt fuses DT but causes asymmetry in IFT which is one of the causes to reduce the efficiency of extraction of IFE (see $[1,2]$ ). To reduce this asymmetry of IFE target, there should be mechanisms to control surface instability. At present, several mechanisms (see [2-6]) have been used to reduce the growth rate of RTI at the interface between heavy fluid supported by a lighter fluid.

In addition to the study of IFE, we note that the interfacial instabilities continue to be the frontier area of research in understanding, control and exploitation of microfluidic devices. In the literature, the work on ordinary Newtonian poorly conducting fluid flows in the presence of an electric field, called Electrohydrodynamics (EHD) (see [7]), flowing through a microchannel has been studied because of their applications in Science, Engineering and Technology, particularly in the effective design of artificial organs in biomedical engineering, solidification process in material science, heat transfer across barriers, friction between surfaces and their mitigation, failure of polymers and so on. Therefore, in biomedical engineering, for an effective design of artificial organ like cartilages in synovial joints, endothelium in Coronary Arteries Disease (CAD) involving poorly conducting fluids, it is necessary to control the growth rate of surface instabilities at the interface between cartilages and synovial fluid in synovial joints (SYJ), at the interface between endothelium and the body fluid in arteries in CAD, at the ablative surface of IFT in IFE. The available literature (see $[8,2]$ ) on surface instabilities is 
mainly concerned with the traditional Newtonian fluid approximations. During the degenerative changes either in synovial joints or in $\mathrm{CAD}$, involving poorly conducting physiological fluids with suspension of nutrients, hyaluronic acid (HA), red blood cells (RBC), white blood cells (WBC), platelets and also in the process of fusing DT in IFE by laser radiation, the Newtonian fluid approximations cannot precisely describe the characteristics of the complex poorly conducting fluids described above. These poorly conducting fluids deform and produce a spin field due to their microrotation forming micropolar fluid developed by Eringen [9]. This micropolar fluid will be more effective in practical problems described above because it takes care of local effects arising from microstructure and as well as the intrinsic motions of microfluidics (see [10-13]). According to Eringen [9], the micropolar fluids may be regarded as non-Newtonian fluids like suspension of DT in IFE target. The spin field due to microrotation of these freely suspended particles like DT in IFE, HA in SYJ and RBC and so on in CAD sets up an antisymmetric stress known as couple stress and thus forming couple stress fluid (CSF). It is according to Eringen [9], a particular case of micropolar fluid, when microrotation balances with the natural vorticity of fluid. This poorly conducting couple stress fluid in the presence of an electric field can be regarded as Electrorheological Fluid (ERF).

We note that the DT in IFT, Synovial Fluid (SF) in SYJ and RBC, WBC and so on in CAD can be regarded as poorly conducting liquid crystals with conductivity, $\sigma$, increasing with the difference in temperature between the higher temperature at the ablative surface of IFT and lower temperature inside the IFE target. This difference in temperature causes difference in conductivity, $\nabla \sigma$. This $\nabla \sigma$ releases the charges from the nuclei forming distribution of charge density, $\rho_{e}$. These charges induce an electric field, $\vec{E}_{i}$ called thermal electric field. If there is a need of higher strength of electric field, we can generate the applied electric field, $\vec{E}_{a}$, by embedding the electrodes of different potentials at the boundaries. The total electric field, $\vec{E}\left(=\vec{E}_{i}+\vec{E}_{a}\right)$ produces, according to Ohm's law, the current density, $\vec{J}=\sigma E_{i}+\rho_{e} q_{i}$, which is the sum of conduction current and convective current and also produces an electric force, $\vec{f}=\rho_{e} \vec{E}$. This current, $\vec{J}$, acts as sensing and the force, $\overrightarrow{f_{e}}$, acts as actuation. These two properties make a poorly conducting couple stress fluid as smart material. This fluid in the presence of an electric filed is called ERF introduced by Winslow [14] and Rajagopal and Wineman [15]. These ERFs have a number of possible technical applications in the various areas of microelectronics, industrial applications in addition to effective design of artificial organs in biomedical engineering and to increase the efficiency of the extraction of IFE as discussed above. Because of their importance considerable interest has been evinced, during the last decade, in the study of their experimental and theoretical point of view (see [15-17]).

The literature on the study of RTI is mainly concerned, as stated above, with ordinary Newtonian fluid. Recently Rudraiah and Chandrashekara [18] have studied the effect of hydrodynamic couple stress fluid, in the absence of electric field, on RTI and showed that this couple stress is very effective in reducing the asymmetry in IFE caused by fusing DT due to laser radiation and in reducing the side effects of haemolysis in biomedical engineering problems discussed above. So far, to our knowledge, much work has not been done on the study of ERTI using smart property of couple stress poorly conducting fluid, regarded as ERF, to reduce the growth rate of ERTI at the ablative surface of IFE target and also in the effective design of artificial organs in biomedical engineering in the presence of an electric filed. The study of it is the main objective of this paper.
To achieve this objective, this paper is planned as follows. The required basic equations and the relevant boundary and surface conditions and the suitable approximations are discussed in Section 2. The electrical conductivity, the conduction temperature in the presence of radiation, electric potential, the electric field and the density of distribution of charges are also obtained in Section 2. Section 3 is devoted to find the velocity distribution. The dispersion relation derived analytically using linear stability analysis in Section 4. The important conclusions are drawn in the final Section 5.

\section{Formulation of the Problem}

We consider a target shell in the form of a thin film of an unperturbed thickness, $h$, filled with an incompressible poorly electrically conducting light couple stress fluid of uniform density, $\rho_{f}$, called region 1 . It is bounded below by a rigid surface and above by an incompressible, poorly conducting couple stress heavy fluid saturated in a densely packed pours layer of large extent $H$ of uniform density, $\rho_{p}$, called region 2 . To generate an applied electric field the lower rigid boundary is embedded with the electrodes of higher potential at $y=0$ and at the interface the electrodes of lower potential are embedded at $y=h$. Here the suffixes $f$ and prefer to the quantities in clear fluid and in porous layer, respectively. The assumption on densely packed fluid saturated porous layer with heavy couple stress poorly conducting fluid is almost static is needed for RTI. The assumption of the nominal surface as in the experimental work of Beavers and Joseph [19] and theoretical work of Rudraiah [20] is needed to maintain laminar flows as well as to use Saffman [21] slip condition. The fluid in the shell is set in motion by acceleration normal to the interface and small disturbances are amplified when the acceleration is directed from the lighter couple stress poorly conducting fluid in the shell to heavy poorly conducting couple stress fluid in the pours layer of large extent $H$ compared to thin film of thickness $h$. This instability at the interface, by definition, is RTI. To investigate this RTI, we consider a rectangular coordinate system $(x, y)$ as shown in Fig. 1 with $x$-axis parallel to the film and $y$-axis normal to it. Here $\eta(x, t)$ is the perturbed interface between the couple stress poorly conducting fluid in the shell and with the porous layer (see Fig. 1).

For the sake of clarity we first give general form of modified basic equations for a poorly conducting couple stress two dimensional incompressible fluid flow, modification in the sense of addition of electric force to be obtained from the general form of Maxwell equations.

The rheological properties of physiological fluids like synovial fluid in synovial joints, blood in arteries reveal that viscosity varies non-linearly with concentration of suspended particles exhibiting either shear thinning or shear thickening behavior. This is one of the non-Newtonian fluid flow properties, which have been studied in the literature (see $[22,23]$ ) but their work is silent about micromotions, microrotation and deformations. These are taken into account in this paper using couple stress fluid as a particular case of "micropolar fluid theory" developed by Eringen [9] as explained in Section 1 above. The basic equations for this fluid are:

Conservation of mass for an incompressible fluid:

$\frac{\partial q_{i}}{\partial x_{i}}=0$

Conservation of linear momentum:

$\rho_{f} \frac{D q_{i}}{D t}=\frac{\partial \tau_{i j}}{\partial x_{j}}+\pi_{i}+\rho_{f} f_{i}+\rho_{e} E_{i}$ 


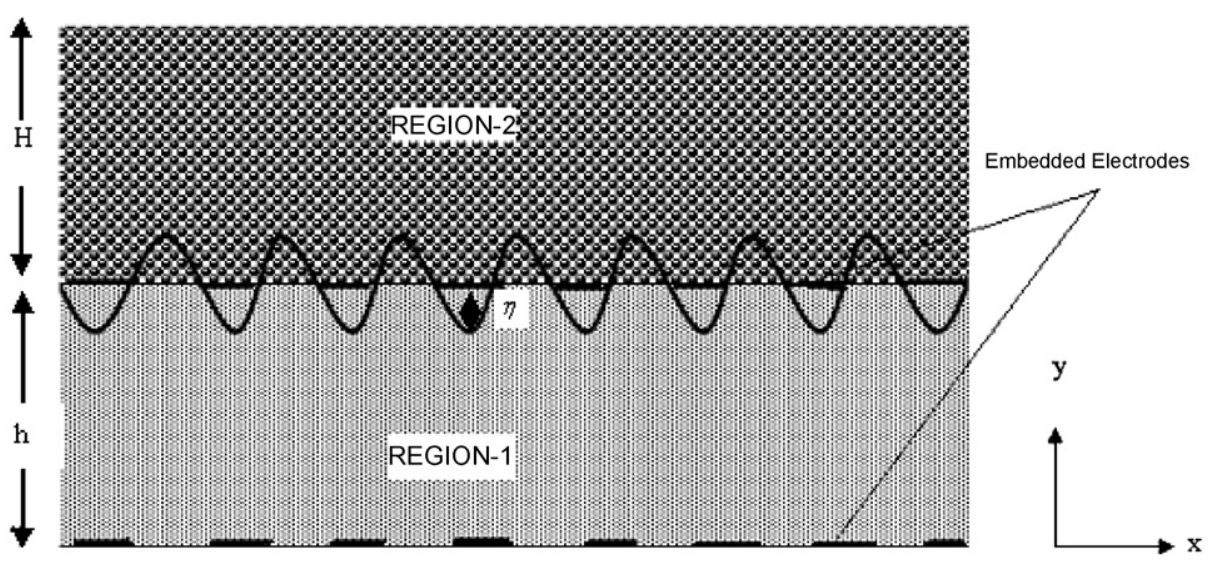

Fig. 1. Physical Configuration.

Conservation of angular momentum:

Angular momentum is usually defined as the moment of the linear moment. If $r_{i}$ is the position vector of the particles and

$p_{i}=\rho q_{i}$

is the linear momentum, then the angular momentum $L_{i}$ is

$$
L_{i}=\varepsilon_{i j k} r_{i} p_{k}
$$

where

$$
\varepsilon_{i j k}=\left\{\begin{array}{lll}
1 & \text { if } & i, j, k \text { take values in cyclic order } \\
-1 & \text { if } & i, j, k \text { take values in acyclic order } \\
0 & \text { if } & \text { two or all of } i, j, k \text { take the same value }
\end{array}\right.
$$

is the Levi-Civita symbol.

The conservation of angular momentum, neglecting the body couple and contact couples, following Rudraiah [23], can be obtained by taking the cross product of $r_{i}$ with Eq. (2) and using Eq. (3) in the form

$\varepsilon_{i j k} r_{i} \frac{\partial p_{k}}{\partial t}+\varepsilon_{i j k} r_{i} \frac{\partial q_{j}}{\partial x_{k}} p_{k}=\varepsilon_{i j k} r_{i} \frac{\partial \tau_{j k}}{\partial x_{k}}+\varepsilon_{i j k} \rho_{f} r_{i} f_{k}+\rho_{e} r_{i} E_{i}$

In Eqs. (1)-(5), $q_{i}(i=1,2)$ is the velocity vector having the components $(u, v), \tau_{j k}$ the stress tensor, $\rho_{f} f_{k}$ the body force, $\rho_{e} E_{i}$ the electric force, $\rho_{e}$ the distribution of charge density and $E_{i}$ is the electric field.

The conservation of charges for incompressible fluid:

$\frac{\partial \rho_{e}}{\partial t}+q_{i} \frac{\partial \rho_{e}}{\partial x_{i}}+\frac{\partial J_{i}}{\partial x_{i}}=0$

where $J_{i}$ is the conduction current density given by

$J_{i}=\sigma E_{i}$

The Maxwell field equations are Gauss law is

$\frac{\partial E_{i}}{\partial x_{i}}=\frac{\rho_{e}}{\varepsilon_{o}}$

where $\varepsilon_{o}$ is the dielectric constant for free space.

In a poorly conducting fluid the induced magnetic field is negligible and there is no applied magnetic field, hence the Faradays law becomes

$\frac{\partial E_{i}}{\partial x_{j}}-\frac{\partial E j}{\partial x_{i}}=0$
That is, the electric field is conservative, so that

$$
E_{i}=-\frac{\partial \phi}{\partial x_{i}}
$$

where $\phi$ is the electric potential.

In a poorly conducting fluid since $\sigma \ll 1$, any perturbation on it is negligible and increases with the conduction temperature, $T_{b}$, such that

$\sigma=\sigma_{o}\left[1+\alpha_{h}\left(T_{b}-T_{o}\right)\right]$

where $T_{o}$ is the atmospheric temperature, $\sigma_{o}$ that of $\sigma$ at $T=T_{o}$, and $\alpha_{h}$ is the volumetric expansion coefficient of $\sigma$.

We note that the limitations encountered in the continuum theory are the lack of taking into account the microrotation of a fluid, for example hyaluronic acid (HA) molecules present in synovial fluid. In that case, the intrinsic motions of the microelements must be taken into account in deriving the required constitutive equations where the microelement motions and deformations play a significant role. In such situations, the Eringen's [9] "micropolar fluid theory" explained in Section 1 above is useful and he showed that the couple stress theory results as a special case of micropolar fluid theory if the microrotation vector is constrained equal to the fluid bulk vorticity throughout the flow field. Then, the constitute equations for couple stress fluid, following Stokes [24] as in Rudraiah [23], are:

$\tau_{i j}=\left(-p+\mu^{\prime} e_{k k}\right) \delta_{i j}+2 \mu e_{i j}$

$\tau_{i j}^{\prime}=-2 \lambda \Omega_{i j, k k}-\frac{\rho}{2} \varepsilon_{i j s} G_{s}$

$M_{i j}=4 \lambda \Omega_{j, i}+4 \lambda^{\prime} \Omega_{i, j}$

where

$e_{i j}=\frac{1}{2}\left(\frac{\partial q_{i}}{\partial x_{j}}+\frac{\partial q_{j}}{\partial x_{i}}\right)$ is the strain tensor

$\Omega_{i}=\varepsilon_{i j k} q_{k, j}=\frac{1}{2}\left(\frac{\partial q_{i}}{\partial x_{j}}-\frac{\partial q_{j}}{\partial x_{i}}\right)$ is the vorticity tensor

$G_{s}$ is the angular velocity vector and $M_{i j}$ is the body moment. Here the dimensions of $\mu^{\prime}$ and $\mu$ are those of viscosity and the couple stress parameters $\lambda$ and $\lambda^{\prime}$ are those of momentum. The ratio $(\lambda / \mu)$ has the dimensions of length squared.

For an incompressible fluid, in the absence of the body moments, the basic equations of motion for couple stress fluid, 
using Eqs. (5), (12)-(14), following Rudraiah [23], are

$\rho_{f}\left(\frac{\partial q_{i}}{\partial t}+q_{i} \frac{\partial q_{i}}{\partial x_{j}}\right)=-\frac{\partial p}{\partial x_{i}}+\mu \frac{\partial^{2} q_{i}}{\partial x_{i}^{2}}-\lambda \frac{\partial^{4} q_{i}}{\partial x_{i}^{4}}+\rho_{e} E_{i}$

The conservation of energy, in the presence of laser radiation, in regions 1 and 2, are:

Region 1: For clear fluid

$$
\frac{\partial T_{f}}{\partial t}+q_{i} \frac{\partial T_{f}}{\partial x_{j}}=K_{f} \frac{\partial^{2} T_{f}}{\partial x_{i}^{2}}+I_{o} \Omega e^{-\Omega y}
$$

Region 2: For porous layer

$$
\frac{\partial T_{p}}{\partial t}+q_{i} \frac{\partial T_{p}}{\partial x_{j}}=K_{p} \frac{\partial^{2} T_{p}}{\partial x_{i}^{2}}+I_{o} \Omega e^{-\Omega y}
$$

where $T_{f}$ and $T_{p}$ are the temperatures in clear fluid and in fluid saturated porous layer, respectively, $\Omega$ is the absorption frequency, $K_{f}$ and $K_{p}$ are the thermal diffusivities of fluid in thin shell and in porous layer, respectively. In region 2 , the poorly conducting couple stress fluid is very heavy and densely packed porous layer so that we assume it as almost static and has uniform density and temperature distribution. From the energy equation, by assuming the fluid in a porous layer is static and is in thermal equilibrium, we get

$\frac{d T^{2} b}{d y^{2}}=-\frac{I_{o} \Omega e^{-\Omega y}}{K}$

The boundaries are assumed to be conducting and isothermal so that we have the boundary conditions:

$T_{b}=T_{o}$ at $y=0$

$T_{b}=T_{1}$ at $y=1$

Solving Eq. (20) using conditions (21) and (22) and using Eq. (11) with the assumption that the absorption frequency $\left(\Omega \cong 10^{6} / \mathrm{m}\right)$ of laser radiation has to decay in a short distance of order $1 / \Omega$, we get,

$\sigma=\sigma_{0}\left[1+\alpha_{h} \Delta T y\right] \cong \sigma_{0} e^{\alpha y}$

where

$\alpha=\alpha_{h} \Delta T$ and $\Delta T=T_{1}-T_{0}$

Further, we assume the frequency of charge distribution is smaller than the corresponding relaxation frequency of the electric field so that the convective current $\rho_{e} q_{i}$ and the time derivative of $\rho_{e}$ are negligible compared to $\nabla \cdot(\sigma E)$, in Eq. (6). Then we get

$\frac{\partial^{2} \phi}{\partial x^{2}}+\frac{\partial^{2} \phi}{\partial y^{2}}+\alpha \frac{\partial \phi}{\partial y}=0$

Satisfying the boundary conditions,

$\phi=\frac{V x}{h}$ at $y=0$

$\phi=\frac{V\left(x-x_{0}\right)}{h}$ at $y=h$

These conditions arise due to the embedded electrodes of different potentials at $y=0$ and $h$ as shown in Fig. 1 and permits a linear variation of $\phi$ with respect to $x$.

Solving Eq. (25), using conditions (26) and (27), after making them dimensionless using the scales $V$ for potential and $h$ for length, we get

$\phi=x-\frac{x_{o}\left(1-e^{-\alpha y}\right)}{\left(1-e^{\alpha}\right)}$ with this, using Eqs. (7)-(10), we get,

$E_{x}=-\frac{\partial \phi}{\partial x}=-1$

$E_{y}=-\frac{\partial \phi}{\partial y}=\frac{x_{0} \alpha e^{-\alpha y}}{1-e^{-\alpha}}$

and

$\rho_{e}=-\varepsilon_{o} \frac{\partial^{2} \phi}{\partial y^{2}}=-\frac{x_{o} \varepsilon_{o} \alpha^{2} e^{-\alpha y}}{1-e^{-\alpha}}$

From these equations we obtain,

$\rho_{e} E_{x}=\frac{x_{o} \varepsilon_{o} \alpha^{2} e^{-\alpha y}}{1-e^{-\alpha}}$

and

$\rho_{e} E_{y}=-\frac{\varepsilon_{o} x_{o}^{2} \alpha_{o}^{3} e^{-2 \alpha y}}{\left(1-e^{-\alpha}\right)^{2}}$

\section{The solution of the problem}

In this section we obtain the velocity and temperature distributions by using the suitable boundary and surface conditions:

\subsection{Velocity Distribution}

To study the problem of RTI posed in this paper, we consider the steady and unidirectional flow of lighter couple stress poorly conducting fluid in region 1 accelerating the heavy dense couple stress poorly conducting fluid saturated in densely packed porous layer in region 2 in the presence of laser radiation with $\eta(x, t)$ as the elevation of the interface at $y=h$. To obtain the required equations, following Rudraiah [23], we use the following combined lubrication and stokes approximations:

1. The thickness $h$ of the film is much smaller than the thickness $H$ of densely packed fluid saturated porous layer above the film, that is

$h \ll H$

2. The non-dimensional Strouhal number, $S$, which is the measure of the local acceleration to the inertial acceleration, is $S=L / U T \ll 1$

Here $U=(v / L)$ the characteristic velocity, $v=(\mu / \rho)$ the kinematic viscosity, $L=\sqrt{\gamma / \delta}$ the characteristic length, $\gamma$ the surface tension, $\delta=g\left(\rho_{p}-\rho_{f}\right)$ and $T=\left(\mu \gamma / h^{3} \delta^{2}\right)$ is the characteristic time scale. These assumptions enabled us to neglect the local acceleration term $\partial q / \partial t$ in Eq. (17).

3. We consider high viscous couple stress fluid so that the inertial acceleration term in Eq. (17) can be neglected compared with the viscous term.

4. The interface elevation $\eta$ is assumed to be small compared with film thickness $h$, that is

$\frac{\eta}{h} \ll 1$

These assumptions enabled us to use the creeping flow approximations which allow us to neglect certain terms in the perturbation equations to obtain linear equations for the interface elevation. Under these assumptions, for two dimensional flow, the basic Eqs. (17) and (1) respectively, using Eqs. (32) and (33) and making them dimensionless using the scales, $h$ for length, $\delta h$ for 
pressure, $\delta h^{2} / \mu_{f}$ for velocity, $\mu \gamma / h^{3} \delta^{2}$ for time, $V / h$ for electric field, $\varepsilon_{o} V / h$ for density of the charges, $\theta_{f, p} T_{o}$ for temperatures, $V$ for electric potential, take the form

$0=-\beta^{2} \frac{\partial p}{\partial x}+\beta^{2} \frac{\partial^{2} u}{\partial y^{2}}-\frac{\partial^{4} u}{\partial y^{4}}+\frac{w_{e} \beta^{2} \alpha_{0}^{2} x_{0} e^{-\alpha y}}{\left(1-e^{-\alpha}\right)}$

$0=-\frac{\partial p}{\partial y}-\frac{w_{e} x_{0}^{2} \alpha^{3} e^{-2 \alpha y}}{\left(1-e^{-\alpha}\right)^{2}}$

$0=\frac{\partial u}{\partial x}+\frac{\partial v}{\partial y}$

where $\beta^{2}=\left(\mu_{f} h^{2} / \lambda\right)$ is the couple stress parameter, $w_{e}=\left(\varepsilon_{0} V^{2} \alpha^{2} x_{0} / \delta h^{2}\right)$ is the electric number which represents physically the measures the electric energy to kinetic energy of the system.

These equations have to be solved using the following nondimensional form of the boundary and surface conditions on velocity:

The no-slip boundary conditions at the rigid surface are

$u=0$ and $v=0$ at $y=0$

The Saffman [21] slip condition at the interface between porous layer and clear fluid layer is

$\frac{\partial u}{\partial y}=-\alpha_{1} \sigma_{p} u$ at $y=1$

where $\sigma_{p}=h / \sqrt{k}$ is the porous parameter and $\alpha_{1}$ is the slip coefficient.

The couple stress boundary conditions are

$\frac{\partial^{2} u}{\partial y^{2}}=0$ at $y=0$ and $y=1$

The dynamic surface condition, at the interface, is

$p=-\eta\left(\delta(1)-w_{e}\right)-\frac{1}{B} \frac{\partial^{2} \eta}{\partial x^{2}}$ at $y=1$

where $B=\delta h^{2} / \gamma$ is the Bond number and $\delta(1)=\theta_{f}(1)-\theta_{p}(1)$.

The kinematic surface condition, at the interface, is

$v=\frac{\partial \eta}{\partial t}+u \frac{\partial \eta}{\partial x}$ at $y=1$

For the linear case the above condition (43) reduces to the form

$v=\frac{\partial \eta}{\partial t}$ at $y=1$

Solution of Eq. (36), satisfying conditions (39)-(41), is

$$
\begin{aligned}
u= & C \cosh \beta y+D \sin h \beta y+\frac{y^{2}}{2} \frac{\partial p}{\partial x}-\frac{A y}{\beta^{2}} \\
& +\frac{w_{e} \beta^{2} x_{o} e^{-\alpha y}}{\left(\alpha^{2}-\beta^{2}\right)\left(1-e^{-\alpha}\right)}+\frac{1}{\beta^{2}}\left(\frac{\partial p}{\partial x}-B_{o}\right)
\end{aligned}
$$

where

$$
\begin{aligned}
& A=\frac{\beta^{2}}{\left(1+\alpha_{1} \sigma_{p}\right)}\left\{a_{1} \frac{\partial p}{\partial x}-\frac{w_{e} x_{0} \alpha^{2} a_{2}}{\left(1-e^{-\alpha}\right)\left(\alpha^{2}-\beta^{2}\right)}+w_{e} x_{0} \alpha_{1} \sigma_{p}\right\}, \\
& a_{1}=\frac{\left(2+\alpha_{1} \sigma_{p}\right)}{2}-\frac{(\cosh \beta-1)}{\beta \sin h \beta}, \quad a_{2}=\frac{\beta\left(e^{-\alpha} \cosh \beta-1\right)}{\sin h \beta}+\frac{\beta^{2} e^{-\alpha}}{\alpha^{2}}, \\
& B_{0}=\frac{-w_{e} x_{0} \beta^{2}}{1-e^{-\alpha}}, \quad C=-\frac{1}{\beta^{2}}\left[\frac{\partial p}{\partial x}+\frac{w_{e} \beta^{2} x_{0} \alpha^{2}}{\left(\alpha^{2}-\beta^{2}\right)\left(1-e^{-\alpha}\right)}\right] \text { and } \\
& D=-\frac{1}{\beta^{2}}\left[\frac{(1-\cosh \beta)}{\sin h \beta} \frac{\partial p}{\partial x}+\frac{w_{e} \beta^{2} x_{0} \alpha^{2}\left(e^{-\alpha}-\cos h \beta\right)}{\left(\alpha^{2}-\beta^{2}\right)\left(1-e^{-\alpha}\right) \sinh \beta}\right]
\end{aligned}
$$

\subsection{Temperature Distribution}

To find the effect of laser radiation on heat transfer in a thin film past a porous layer, we assume, as in Section 3.1, fully developed flow with basic state as $\vec{q}=(u(y), 0), T_{f, p}=T_{f, p}(y)$, and making Eqs. (18) and (19) dimensionless using the scales mentioned in Section 3.1, we get,

$0=\frac{\partial^{2} \theta_{f}}{\partial y^{2}}+s_{f} e^{-\Omega y}$

$0=\frac{\partial^{2} \theta_{p}}{\partial y^{2}}-s_{p} e^{-\Omega y}$

where $s_{f}=\left(I_{o} \Omega h^{3} / T_{o} k_{f}\right)$ and $s_{p}=\left(I_{o} \Omega h^{3} / T_{o} k_{p}\right)$.

To find the solutions of Eqs. (46) and (47), we consider the following two cases for a poorly conducting fluid in porous layer.

Case 1: This fluid to be homogeneous.

Case 2: This fluid to be Boussinesq fluid.

For a poorly conducting couple stress homogeneous incompressible fluid in thin-film and in porous layer, we assume that the temperature, $T_{p}$, in the porous layer is uniform. Then Case 1 : The boundary conditions on temperature for this case are:

Set 1 :

$\theta_{f}=1$ at $y=0$
$\theta_{f}=\theta_{1}$ at $y=1$

Set 2:

$\theta_{f}=1$ at $y=0$

$\frac{\partial \theta_{f}}{\partial y}=-B_{i}\left(\theta_{b}-1\right)$ at $y=1$

where $B_{i}=\left(h_{c} h / k_{f}\right)$, is the Biot number and $\theta_{f}=\theta_{b}$ at $y=1$.

Solving Eq. (46) using set 1 boundary conditions (48) and (49), we get

$\theta_{f}=\frac{s_{f}}{\Omega^{2}}\left(1-e^{-\Omega y}\right)+\frac{s_{f}\left(e^{-\Omega}-1\right)}{\Omega^{2}} y+\left(\theta_{1}-1\right) y+1$

In this case

$\delta(1)=\theta_{b}-\theta_{p}$

where $\theta_{p}$ is the constant temperature of the fluid saturated porous layer.

Case 2: The boundary conditions on temperature, for Boussinesq fluid in the thin film as well as in the porous layer, are

$\theta_{f}=1$ at $y=0$

$\frac{\partial \theta_{f}}{\partial y}=-B_{i}\left(\theta_{f}-1\right)$ at $y=1$

$\frac{\partial \theta_{p}}{\partial y}=-B_{i}\left(\theta_{p}-1\right)$ at $y=1$

$\frac{\partial \theta_{p}}{\partial y} \rightarrow 0$ as $y \rightarrow \infty$

The solutions of Eqs. (46) and (47) using the this boundary conditions (53)-(56) are

$\theta_{f}=1-\frac{s_{f}\left(e^{-\Omega y}-1\right)}{\Omega^{2}}+\frac{B_{i} s_{f}\left(e^{-\Omega}-1\right)}{\Omega^{2}\left(B_{i}+1\right)} y-\frac{s_{f} e^{-\Omega}}{\Omega\left(B_{i}+1\right)} y$ 
and

$\theta_{p}=1+\frac{s_{p}}{\Omega^{2}} e^{-\Omega y}+\frac{s_{p} e^{-\Omega}}{\Omega^{2} B_{i}}\left(B_{i}-\Omega\right)$

Now using Eqs. (57) and (58), we obtain an expression for $\delta(1)$ in the following form:

$\delta(1)=\theta_{f}(1)-\theta_{p}(1)$

That is

$\delta(1)=\frac{s_{f}}{\Omega^{2}}\left[\frac{1-(1+\Omega) e^{-\Omega}}{B_{i}+1}\right]-\frac{s_{p} e^{-\Omega}\left(2 B_{i}-\Omega\right)}{\Omega^{2} B_{i}}$

\section{Dispersion Relation}

Integrating Eq. (38) over the limits 0 to 1 and using conditions (39), we get

$v(1)=-\int_{0}^{1} \frac{\partial u}{\partial x} d y$

This, using Eq. (45) and integrating, we get

$v(1)=\left\{\frac{1}{3}-\left[\frac{4+\beta^{2}}{4 \beta^{2}}-\frac{2(\cos h \beta-1)}{\beta^{3} \sin h \beta}+\frac{2(\cosh \beta-1)-\beta \sin h \beta}{4 \beta \sinh \beta\left(1+\alpha_{1} \sigma_{p}\right)}\right]\right\} \frac{\partial^{2} p}{\partial x^{2}}$

From Eq. (44), using the normal mode solution of the form $\eta=\eta_{0} e^{i l x+n t}$ and using Eqs. (42) and (62), we get the dispersion relation in the form

$n=\left\{\frac{1}{3}-\left[\frac{4+\beta^{2}}{4 \beta^{2}}-\frac{2(\cos h \beta-1)}{\beta^{3} \sin h \beta}+\frac{2(\cos h \beta-1)-\beta \sin h \beta}{4 \beta \sin h \beta\left(1+\alpha_{1} \sigma_{p}\right)}\right]\right\} l^{2}\left(\delta(1)-w_{e}-\frac{l^{2}}{B}\right)$

where $n$ is the growth rate in the presence of couple stress, electric field and laser radiation. In the absence of electric field, couple stress, porous layer and laser radiation that is as $w_{e} \rightarrow 0$, $\lambda \rightarrow 0(\beta \rightarrow \infty), \sigma_{p} \rightarrow 0$ and $\delta(1) \rightarrow 1$, the dispersion relation (63) reduces to

$n_{b}=\frac{l^{2}}{3}\left(1-\frac{l^{2}}{B}\right)$

which coincides with the expression given by Babchin et al. [25]. Then Eq. (63) can be written as

$n=n_{b}-\left[\frac{4+\beta^{2}}{4 \beta^{2}}-\frac{2(\cosh \beta-1)}{\beta^{3} \sin h \beta}+\frac{2(\cosh \beta-1)-\beta \sinh \beta}{4 \beta \sin h \beta\left(1+\alpha_{1} \sigma_{p}\right)}\right] l^{2}\left(\delta(1)-w_{e}-\frac{l^{2}}{B}\right)$

\section{Results and Conclusions}

The linear ERTI of an incompressible viscous poorly conducting couple stress fluid in a thin film in the presence of an electric filed and laser radiation, bounded below by a rigid surface and above by densely packed porous layer consisting of heavy couple stress poorly conducting fluid of high density is studied using linear stability analysis combined with the normal mode solution. The dispersion relation given by Eq. (65) following [8,2] can be expressed in the form:

$n=n_{b}-\beta^{*} l v_{a}$

where

$v_{a}=\left[\frac{4+\beta^{2}}{4}-\frac{2(\cosh \beta-1)}{\beta \sinh \beta}+\frac{2 \beta(\cosh \beta-1)-\beta \sinh \beta}{4 \sinh \beta\left(1+\alpha_{1} \sigma_{p}\right)}\right] l\left(\delta(1)-w_{e}-\frac{l^{2}}{B}\right)$ is the transverse velocity at the interface, $\beta^{*}=\left(1 / \beta^{2}\right), n_{b}$ is given by Eq. (64).

Setting $n=0$ in the dispersion relation (63), we get the critical wave number, $l_{c t}$, as

$l_{c t}=\sqrt{\left(\delta(1)-w_{e}\right) B}$

The maximum wave number, $l_{m}$, obtained from Eq. (63), by setting $(\partial n / \partial l)=0$ is

$l_{m}=\frac{l_{c t}}{\sqrt{2}}$

Relations (68) and (69) are true even in the absence of couple stress, electric field and laser radiation effects and for convenience we call them as classical results denoted by suffix $b$. Substituting Eq. (69) into Eq. (63), we get the maximum growth rate in the form

$n_{m}=\frac{B\left(\delta(1)-w_{e}\right)^{2}}{4}\left\{\frac{1}{3}-\left[\frac{4+\beta^{2}}{4 \beta^{2}}-\frac{2(\cosh \beta-1)}{\beta^{3} \sinh \beta}+\frac{2(\cosh \beta-1)-\beta \sinh \beta}{4 \beta \sinh \beta\left(1+\alpha_{1} \sigma_{p}\right)}\right]\right\}$

Similarly, using Eqs. (64) and (68), we get the classical maximum growth rate as

$n_{m_{b}}=\frac{B}{12}$

The ratio of the growth rates, $G_{m}=n_{m} / n_{m b}$, is obtained from Eqs. (70) and (71) as

$G_{m}=\left(\delta(1)-w_{e}\right)^{2}\left\{1-3\left[\frac{4+\beta^{2}}{4 \beta^{2}}-\frac{2(\cosh \beta-1)}{\beta^{3} \sinh \beta}+\frac{2(\cosh \beta-1)-\beta \sin h \beta}{4 \beta \sinh \beta\left(1+\alpha_{1} \sigma_{p}\right)}\right]\right\}$

where $\delta(1)$ is given by Eq. (60). We note that in the limit $\lambda \rightarrow 0$ $\left(\beta^{2} \rightarrow \infty\right), w_{e} \rightarrow 0$ and $\delta(1) \rightarrow 1$, this ratio of growth rates given by Eq. (72) reduces to

$G_{m}=\frac{4+\alpha_{1} \sigma_{p}}{4\left(1+\alpha_{1} \sigma_{p}\right)}$

Table 1

Reduction in growth rate for different values $\beta$ Couple stress parameter (For varying electric number $w_{e}$ and slip parameter $\alpha_{1}$ ).

\begin{tabular}{|c|c|c|c|c|c|c|}
\hline$\beta$ & $w_{e}$ & $\delta(1)$ & $\alpha_{1}$ & $\sigma_{p}$ & $G_{m}$ & $\begin{array}{l}\text { Reduction in } \\
\text { growth rate (\%) }\end{array}$ \\
\hline 0.1 & 1 & 0.5 & 0.001 & 10 & 0.00392986 & 99.607 \\
\hline 0.75 & & & & & 0.0116312 & 98.8369 \\
\hline 1.5 & & & & & 0.0399001 & 96.01 \\
\hline 2.25 & & & & & 0.0730293 & 92.6971 \\
\hline 0.1 & 2 & 0.5 & 0.0001 & 10 & 0.00230254 & 99.7697 \\
\hline 0.75 & & & & & 0.10476 & 89.524 \\
\hline 1.5 & & & & & 0.361254 & 63.8746 \\
\hline 2.25 & & & & & 0.661414 & 33.8586 \\
\hline
\end{tabular}

Table 2

Reduction in growth rate for different values $\beta$ couple stress parameter (for varying $\delta(1)$ and porous parameter $\sigma_{p}$ )

\begin{tabular}{lllllll}
\hline$\beta$ & $\mathbf{w}_{\mathbf{e}}$ & $\delta(1)$ & $\alpha_{1}$ & $\sigma_{p}$ & $G_{m}$ & $\begin{array}{l}\text { Reduction in } \\
\text { growth rate (\%) }\end{array}$ \\
\hline 0.1 & 1 & 0.5 & 0.001 & 5 & 0.00115059 & 99.8849 \\
0.75 & & & & & 0.0116228 & 98.8377 \\
1.5 & & & & & 0.0400291 & 95.9971 \\
2.25 & & & & & 0.073283 & 92.6717 \\
0.1 & 1 & 0.75 & 0.001 & \multirow{2}{*}{0.000982464} & 99.9018 \\
0.75 & & & & & 0.00290781 & 99.7092 \\
1.5 & & & & & 0.00997502 & 99.0025 \\
2.25 & & & & & 0.0182573 & 98.1743 \\
\hline
\end{tabular}




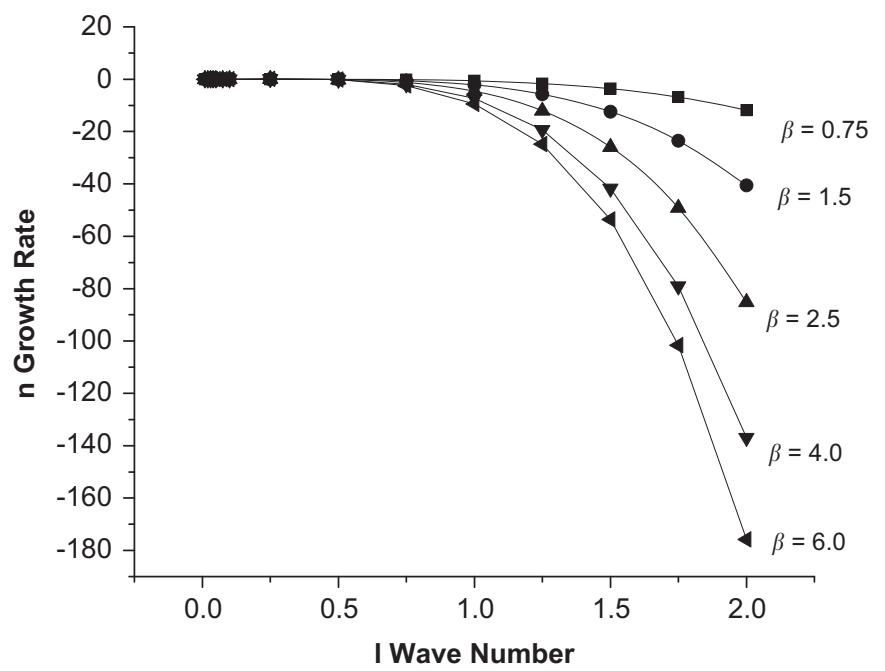

Fig. 2. Graph of $n$ versus 1 for different values of $\beta$.

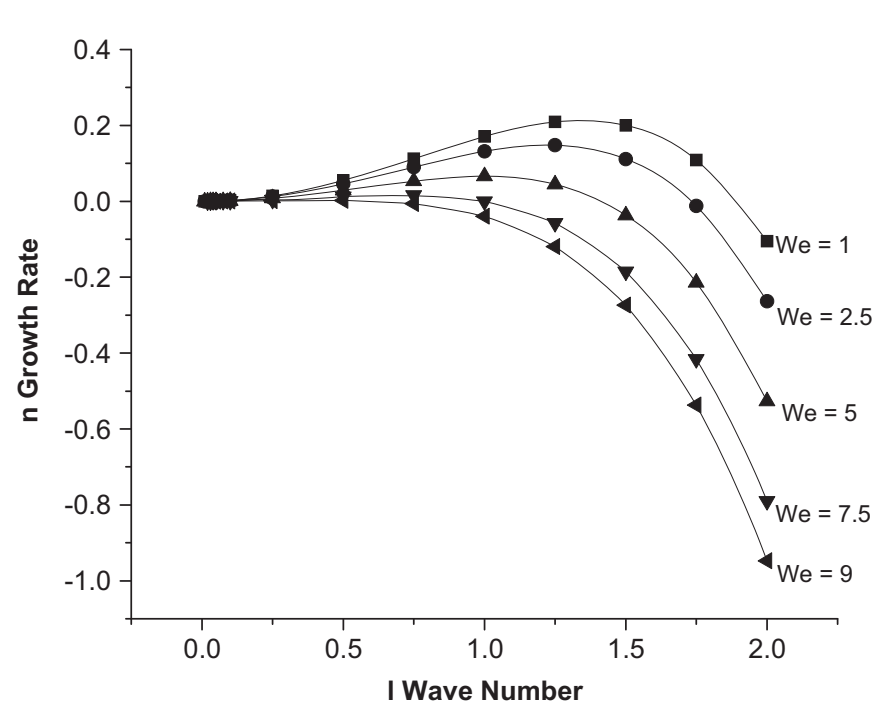

Fig. 3. Graph of $n$ versus 1 for different values of $W_{e}$.

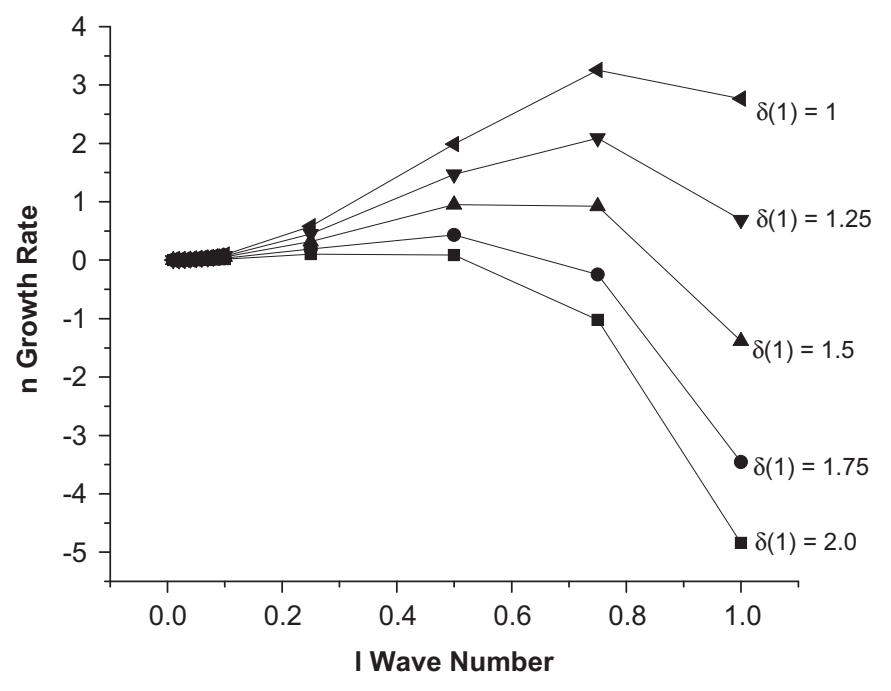

Fig. 4. Graph of $n$ versus 1 for different values of $\delta(1)$.

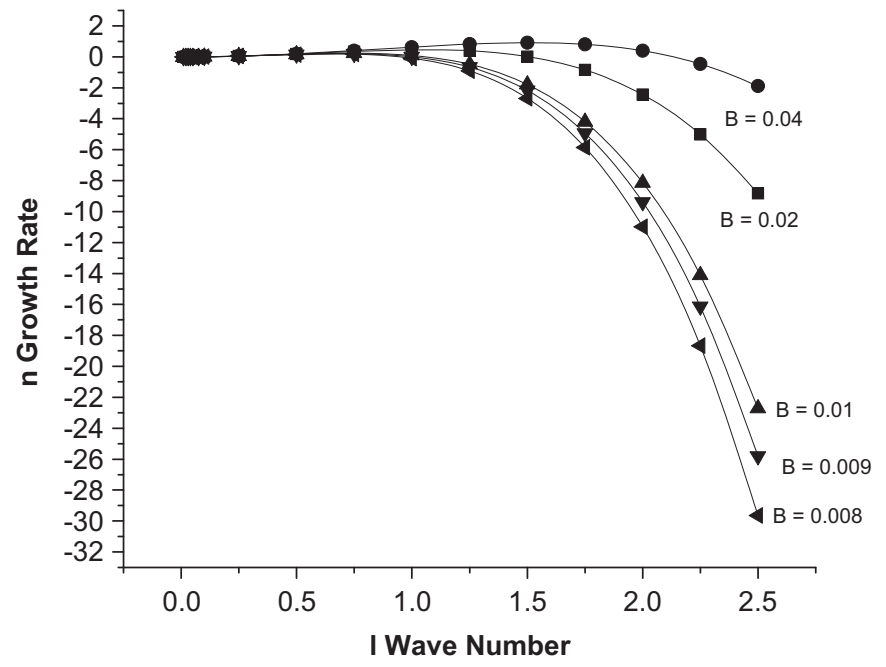

Fig. 5. Graph of $n$ versus 1 for different values of B.

this coincides with the one given by Rudraiah [2] in the absence of couple stress and electric field effects, called NR-formula.

This, $G_{m}$ given by Eq. (72), is computed for different values of the couple stress parameter $\beta$, electric number $w_{e}$, porous parameter $\sigma_{p}$ and absorption frequency $\Omega$ (expressed in difference in temperatures $\delta(1)$ at the interface) at the interface of the laser radiation and the results are tabulated in Tables 1 and 2 .

From these tables, we note that the reduction of growth rate is $99 \%$ in the presence of laser radiation; couple stress and electric field, compared to $45 \%$ reduction predicted by Takabe et al. [8] and $79 \%$ by Rudraiah [2] in the absence of these quantities.

Also the growth rate $n$ given by Eq. (63) is always real. This $n$ is computed for different values of $\beta, w_{e}, B$ and $\delta(1)$ and the values are depicted in Figs. 2-5, these Figs. 2-5 represent the dispersion relation $n$ versus cutoff wave number $l$.

Fig. 2 represents the graph of the growth rate $n$ versus wave number 1 for different values of $\beta$ and with fixed values of $w_{e}=1$, $\delta(1)=10, B=0.02$ and $\sigma=0.001$. This figure shows decrease in $\beta$ (increase in $\lambda$ couple stress parameter) the negative growth rate $n$ decreases. Particularly for $\beta=0.75$ the growth rate $n$ has almost approaches zero and hence stabilizes the interface elevation.

Fig. 3 represents the graph of the growth rate $n$ versus wave number 1 for different values of $w_{e}$ and with fixed values of $\beta=1$, $\delta(1)=10, B=0.02$ and $\sigma=0.001$. This graph shows that for an increase in $w_{e}$ the growth rate $n$ decreases. Particularly for $w_{e}=9$ the growth rate $n$ is very small and hence stabilizes the interface elevation.

Fig. 4 represents the graph of the growth rate $n$ versus wave number 1 for different values of $\delta(1)$ and with fixed values of $\beta=1$, $w_{e}=1, B=0.02$ and $\sigma=0.001$. This figure shows that for an increase in $\delta(1)$ the growth rate $n$ decreases. Particularly for $\delta(1)=1$ the growth rate $n$ is very small and hence stabilizes the interface elevation.

Fig. 5 represents the graph of the growth rate $n$ versus wave number 1 for different values of Bond number $B$ with fixed values of $\beta=1, w_{e}=1, \delta(1)=10$ and $\sigma=0.001$. This figure shows that decrease in $B$ (increase in surface tension) increases the negative growth rate. Particularly for $B=0.04$ the growth rate $n$ is very small and hence stabilizes the interface elevation.

Finally, from these graphs, we predict that the reduction of maximum growth rate in presence of an electric field, laser radiation and couple stress. Hence, we conclude that the electric field, laser radiation and couple stress are more effective in reducing the asymmetry of IFE caused by fusing DT by laser 
radiation compared to classical growth rate in the absence of electric field and couple stress parameter. This conclusion on the reduction of growth rate of the ERTI mode is more effective in the design of a suitable IFT for increasing the efficiency of extraction of IFE by reducing the asymmetry caused by laser radiation. These conclusions are also useful in the effective design of artificial organs in biomedical engineering to prevent the side effects like heamolysis.

\section{Acknowledgement}

This work is supported by ISRO under the research project nos. ISRO/RES/2/338/2007-08 and ISRO/RES/2/335/2007-08. ISRO's financial support to carry out this work is gratefully acknowledged. One of us (G. Chandrashekara) gratefully acknowledges ISRO for providing JRF to carry out this research work.

\section{References}

[1] S. Nakai, K. Mima, Laser driven IFE, present and prospective, Rep. Prog. Phys. 67 (2003) 321.

[2] N. Rudraiah, Effect of porous lining on reducing the growth rate of RayleighTaylor instability in the inertial fusion energy target, fusion Sci. Technol. 43 (2003) 307-311.

[3] T. Desai, H.C. Panth, control of RTI in laser accelerated seeded target Laser part beam, 18(2000)119.

[4] N.G. Boriesenko, Yu.A. Merkuiev, Preprint PN labde, Physics Institute, Moscow, 1990.

[5] K.O. Mikelian, Effect of viscosity on RT and M instabilities, Phys. Rev. E 47 (1993) 375.

[6] N. Rudraiah, B.S. Krishnamurty, R.D. Mathad, The effect of oblique magnetic field on the surface instability of finite conducting fluid layer, Acta, Mech. 119 (1996) 165.
[7] N. Rudraiah, M. Kalal, Electrohydrodynamic surface instabilities; role of porous lining at the alative surface of laser-driven inertial fusion energy target, Current Sci. 93 (5) (2007) 628-647.

[8] H. Takabe, K. Mima, L. Montierth, R.L. Morse, Self consistent growth rate of Rayleigh-Taylor instability in an ablatively accelerating plasma, Phys. Fluids 28 (12) (1985) 3676.

[9] A.C. Eringen, Theory of micropolar fluids, J. Math. Mech. 16 (1966) 1-18.

[10] J. Peddieson, R.P. McNitt, Boundary layer theory for a micropolar fluid, Recent Adv. Eng. Sci. 5 (1970) 405-476.

[11] T. Ariman, M.A. Truk, N.D. Sylvester, Microcontinuum fluid mechanics-a review, Int. J. Eng. Sci. 11 (1973) 905-930.

[12] G. Lukaszewicz, Micropolar Fluids: Theory and Application, Birkhauser, Basel, 1999.

[13] A.C. Eringen, Microcontinuum Field Theories. II: Fluent Media, Springer, NewYork, 2001.

[14] W.M. Winslow, Induced fibration of suspensions, J. Appl. Phys. 20 (1949) 1137-1140.

[15] K. Rajagopal, A.S. Wineman, On constitutive equations for electrorheological materials, Continum Mech. Thermodyn. 7 (1995) 1-22.

[16] K. Rajagopal, M. Ruzicka, On the modeling of electrorheological materials, Mech. Res. Commun. 23 (1996) 401-407.

[17] M. Ruzicka, A note on steady flow of fluids with shear dependent viscosity Nonlinear Anal. 30 (1997) 3029-3039.

[18] N. Rudraiah, G. Chandrashekara, Effects of couple stress on the growth rate of Rayleigh-Taylor instability at the interface in a finite thickness couple stress fluid, J. Appl. Fluid Mech. 3 (1) (2010) 83-89.

[19] G.S. Beavers, D.D. Joseph, Boundary conditions at naturally permeable wall, J Fluid Mech. (1967) 197-207.

[20] N. Rudraiah, Coupled parallel flows in a channel a boundary porous medium of finite thickness, ASME J Fluid Energy 1, 107 (1985) 322.

[21] P.G. Saffman, On the boundary conditions at the surface of a porous medium, Stud. Appl. Math. 50 (1971) 93-101.

[22] Y.C. Fung, in: "Biomechanics", Springer and Verlag, New York, 1981.

[23] N. Rudraiah, Anatomy and biomechanics of synovial joints-Part II: mathematical modelling, in: I.M. Thomas, K. Srinivasa, T. Rajeshwari, Sayee Rajangam (Eds.), Recent Trends in Basic and Applied Anatomy, Rajiv Gandhi University of Health Science, Karnataka1998, pp. 37-105.

[24] V.K. Stokes, Effects of couple stress in fluid on hydromagnetic channel flow, Phys. Fluids 11 (1968) 1131-1133.

[25] A.J. Babchin, A.L. Frenkel, B.G. Levich, Non-linear saturation of RayleighTaylor instability in a thin film, Phy. Fluids 26 (1983) 3159. 